\title{
¿De Política de Representación a Política de Coalición? Posibilidades de Movilización Feminista en el Chile Post-Dictadura
}

\author{
Claudia Mora* \\ Marcela Ríos**
}

\begin{abstract}
Resumen: La historia del movimiento feminista chileno demuestra que independiente de la permanencia de condiciones de desigualdad de género en el tiempo, la acción colectiva emerge como producto de la apertura de estructura de oportunidades y de la agencia de un grupo articulado de activistas. Los movimientos de mujeres en Chile han surgido en el marco de estas condiciones, disolviéndose luego de la consecución del propósito unificador. En este trabajo planteamos que la estructura de oportunidades para la acción feminista se ha abierto bajo la administración Bachelet, pero que la política de la representación característica de los movimientos históricos no es la única -ni la más efectiva- alternativa para avanzar demandas de género. Un análisis de la fragmentación de los movimientos de mujeres post-dictadura indica la necesidad de atender al problema que plantea para los movimientos sociales el forjamiento de una identidad que represente al grupo. En este trabajo sostenemos que una política de representación debe dar paso a una política de coalición, no sólo por sus posibilidades democráticas, sino que también porque en el nuevo marco socio-cultural, la conformación de coaliciones en torno a demandas de género es la vía más efectiva hacia la transformación del orden de género.
\end{abstract}

Palabras clave: movimiento feminista, estructura de oportunidades, política de coalición

\section{From Policy of Representation to Policy of Coalition? Possibilities of Feminist Mobilization in Post-Dictatorship Chile}

\begin{abstract}
The history of the Chilean feminist movement shows that regardless of the permanency of gender inequality collective action emerges when both, the opening of political opportunity structures and the agency of activists converge. Women's movements in Chile have materialized when both of these conditions are present, ceasing to exist after achieving their immediate unifying goal. In this work, we propose that opportunity structures for feminist action have opened under the Bachelet administration but that the politics of representation, historically characteristic of social movements, is no longer the only -or more
\end{abstract}

\footnotetext{
*Universidad Alberto Hurtado, Santiago, Chile. Email:cmora@uahurtado.cl

** PNUD, Santiago, Chile. Email: marcela.rios@undp.org
} 
effective- way to move forward gender demands. An analysis of the fragmentation of women movements points to the need to think about the issue of identity politics in social movements. In this article we suggest that a politics of representation should give way to a politics of coalition, not only for its democratic possibilities but also because in the new socio-cultural context, the articulation of coalitions around gender issues is the most effective alternative to affect the transformation of the gender order.

Key words: feminist movement, opportunities structure, politics of coalition

\title{
¿De Política de Representação a Política de Coalizão? Possibilidades de Mobilização Feminista no Chile Pós-Ditadura
}

\begin{abstract}
Resumo: A história do movimento feminista chileno demonstra que independente da permanência de condições de desigualdade de gênero no tempo, a ação coletiva emerge como produto da abertura de estrutura de oportunidades e da agência de um grupo articulado de ativistas. Os movimentos de mulheres em Chile surgiram no marco destas condições, dissolvendo-se depois da consecución do propósito unificador. Neste trabalho propomos que a estrutura de oportunidades para a ação feminista se abriu baixo a administração Bachelet, mas que a política da representação característica dos movimentos históricos não é a única -nem a mais efetiva- alternativa para avançar demandas de gênero. Uma análise da fragmentação dos movimentos de mulheres pós-ditadura indica a necessidade de atender ao problema que propõe para os movimentos sociais o forjamiento de uma identidade que represente ao grupo. Neste trabalho sustentamos que uma política de representação deve dar passo a uma política de coalizão, não só por suas possibilidades democráticas, senão que também porque no novo marco sócio-cultural, a conformación de coalizões em torno de demandas de gênero é a via mais efetiva para a transformação do ordem de gênero.
\end{abstract} ca de coalizão

Palavras-chave: movimento feminista, estrutura de oportunidades, políti-

Recibido: 26.09.2009

Aceptado: 26.10.2009

$* * *$

\section{Introducción}

Los movimientos sociales, como forma particular de acción colectiva, se fundan en un objetivo común y una solidaridad grupal para plantear desafíos colectivos (Tarrow, 1998). Distintas definiciones de movimiento social postulan que este impugna el poder de las élites de manera sostenida, y que esta demanda está apoyada en una identidad grupal o sentido de pertenencia que se construye en torno a ideales y objetivos en común. Como una forma de acción colectiva, y en su definición clásica, los movimientos de mujeres se refieren a los movimientos organizados en torno una identidad femenina representativa del grupo, y que interactúa con aliados, oponentes y autoridades en el planteamiento de sus reclamaciones (Ríos, 2009). 
Los movimientos de mujeres en Chile han surgido esporádicamente a lo largo del siglo XX, a pesar de la continuidad en la condición de desventaja que las mujeres en cuanto grupo han enfrentado en distintas dimensiones de la vida social. La permanencia del género como uno de los ejes de desigualdad social se ha visto reflejada en las limitaciones históricas a las que las mujeres han estado sujetas, como por ejemplo en el acceso a la educación, al trabajo remunerado, y a derechos ciudadanos. La inequidad producto del sistema de jerarquía social basado en género, se deriva de relaciones sociales institucionalizadas que tienen efectos tanto materiales como simbólicos, pero que son susceptibles de ser desafiadas, impugnadas y rearticuladas por la operación de factores económicos, políticos, o sociales.

Dentro de estos últimos, históricamente ha sido relevante la acción concertada de los movimientos de mujeres como impulsor clave de transformaciones jurídicas, institucionales y simbólicas del orden de género. La movilización de mujeres emergió en Chile en las primeras décadas del siglo XIX, en torno a la confluencia de asuntos privados con el área pública. Ello tiene particular sentido considerando que la vinculación de lo femenino con el espacio doméstico y de cuidado ha sido una de las principales razones históricas de la marginación de las mujeres del espacio público (Valenzuela y Mora, 2009). Sin embargo, no fue sino hasta un siglo más tarde, a comienzos de 1900, que un activismo reconocible surgió ligado a debates de colectivos y clubes de señoras, organizadas en torno a temas como la reforma del Código Civil, educación, trabajo y otros problemas sociales ligados a la pobreza y la salud (Lavrín 1995).

Los movimientos se articularon en base a demandas de género propiamente tal en dos momentos históricos: el primero, reivindicativo de derechos civiles a comienzos del siglo XX; y el segundo, como parte de la reivindicación de la democracia y derechos humanos en los años 70 . Estos momentos muestran que, como argumentan distintos autores, los movimientos emergen cuando los actores perciben patrones de oportunidad política que los incentiva a la acción. En el caso chileno, los movimientos de mujeres se constituyeron en momentos de realineamiento de fuerzas políticas y tomaron fuerza a través de la vinculación a otras luchas sociales. Sin embargo, la permanencia en el tiempo resultó truncada (en especial en el caso de la movilización surgida durante la dictadura militar) por las disputas en torno a los intereses que eran representados por el movimiento.

Estos quiebres hicieron patente que la identidad colectiva refleja diferencias de poder en la definición de asuntos, intereses y estrategias, que a menudo se vierten en conflictos y fragmentación. En ambos movimientos de mujeres chilenas, la permanencia de la acción colectiva se vio debilitada después de la obtención del propósito que los unía simbólicamente (sufragio, en el primer caso; y la consecución de la democracia, en el segundo), por tensiones sobre los intereses representados en la movilización. 
Sin embargo, los movimientos no sólo persiguen como propósito el reconocimiento de reivindicaciones específicas. La acción colectiva puede perseguir la transformación del orden político en el que se mueve, cuestionando las presunciones con las que operan los espacios de poder cuyos supuestos constituyen barreras para el acceso a la participación, ciudadanía, o recursos sociales particulares (Álvarez, Dagnino y Escobar, 1998). Para lograr este propósito, no es imprescindible una política de representación en la que una identidad colectiva se erige como representativa del grupo demandante. En el caso del activismo feminista de la última década en Chile, por ejemplo, los asuntos en torno a los cuales se ha generado debate y un cierto grado de movilización: divorcio, violencia intrafamiliar, derechos reproductivos, participación política, pueden ser entendidos como un cuestionamiento al orden de género, además de como reivindicaciones. Directa e indirectamente, este activismo ha promovido una transformación del poder simbólico de las mujeres, así como la "normalización” de su presencia en la esfera pública (en el trabajo remunerado y la política, por ejemplo). Consecuentemente, el debilitamiento del movimiento de mujeres en Chile post-dictadura no ha significado la ausencia de discusión de los intereses del género ni la disminución de la presión por transformar el orden de género material, institucional y simbólicamente.

En este trabajo planteamos que aún cuando la transformación del contexto socio-cultural y económico ha afectado la posibilidad de configurar un movimiento de mujeres en base a la política de la representación, ello no ha impedido la conformación de alianzas en torno a asuntos de género específicos. De este modo, se puede sostener que la fragmentación del movimiento post-dictadura y la erosión de nociones de solidaridad y acción colectiva en el campo cultural y político han repercutido en un debilitamiento de la protesta y denuncia como estrategia. Pero que, aún cuando ello ha dificultado la emergencia de un objetivo y de una identidad común que mueva la acción colectiva, no ha implicado la clausura del debate y acción feminista. La producción cultural y científica, la diversificación de medios de comunicación de nuevo tipo, así como el surgimiento de una gran variedad de pequeños colectivos a lo largo del país es una muestra de la vitalidad de este accionar.

Si esto es así, ¿es justificada la nostalgia por un movimiento feminista o de mujeres? ¿Es un movimiento de mujeres el actor social privilegiado en la lucha por la transformación del orden de género? Sugerimos que no, y avanzamos la efectividad de una política de coalición como forma paralela de acción social. En las siguientes secciones, proporcionamos un análisis de las estructuras de oportunidades percibidas y utilizadas por los movimientos históricos de mujeres; planteamos que la escisión de los movimientos es un efecto inexorable de la política de la representación; y sugerimos la posibilidad de una efectiva política de coalición en el marco del gobierno Bachelet. 


\section{Movimientos Sociales y Estructura de Oportunidades}

El elemento gatillante en la articulación de un movimiento está dado por condiciones contextuales, en la convergencia de un conjunto interrelacionado de condiciones políticas, además de la agencia de un grupo articulado de activistas. La estructura de oportunidades que se despliega se materializa como producto de la interacción entre los distintos actores, cuyas decisiones están de hecho, determinadas por la percepción de que estas oportunidades están abiertas (Gamson \& Meyer 1996).

La movilización social emerge en la medida que las oportunidades políticas cambian o se adaptan a nuevas condiciones, abriendo posibilidades y generando incentivos para la resistencia. La estructura de oportunidades disponible a los actores, así como las interpretaciones simbólicas e ideológicas que informan sus actos, van a determinar a su vez la manera en que se desarrolla la resistencia (formas de organización, estrategias políticas, tipos de movilización). De este modo, los movimientos sociales son el resultado de la asociación entre estructuras organizacionales, sistema de oportunidades políticas y significado cultural o simbólico. Por ello, no se refiere a una única organización ni a un grupo de redes estructuradas, sino que una red amplia de relaciones sociales e interacciones, un campo de acción donde las personas, individual y colectivamente, adhieren a un conjunto de valores culturales o principios y se involucran en distintos niveles de conexión, tanto entre ellos como con las estructuras sociales y políticas que los rodean (McAdam, McCarthy y Zald 1996). Un movimiento social requiere por tanto aglutinar a un conjunto de organizaciones, individuos, redes de comunicación en torno a un conjunto amplio de demandas colectivas comunes, como por ejemplo la defensa del medio ambiente, de los derechos de igualdad de minorías sexuales, o la igualdad entre mujeres y hombres.

Así, el surgimiento del primer movimiento feminista en Chile en los años 1920-1930, fue posible por la transformación de la estructura de oportunidades políticas que tuvo lugar en la época: por un lado, el crecimiento de luchas sociales se tradujo en definitiva en una amplia lucha por la democratización de la sociedad, lo que creó las condiciones para la movilización pública y la creación de puentes entre este movimiento y las demandas feministas. Por otro, la existencia de un realineamiento de las alianzas político partidistas posibilitó la emergencia de una nueva estructura de alianzas entre sectores progresistas (Drake 1978).

Este primer movimiento social con una agenda de género, emergió en torno a la obtención de derechos ciudadanos, asunto que se constituyó como el baluarte principal de la emergente alianza feminista. La centralidad de la demanda por ciudadanía política sin embargo, no excluía otras demandas y discursos en la medida que las organizaciones de mujeres de esa época tenían un fuerte interés por los problemas sociales de su tiempo, justicia social, condiciones de salud, educación entre otros (Ríos 2009). La obtención del derecho a voto en elecciones municipales proporcionó un 
impulso a la organización del movimiento por el derecho a sufragio general, que finalmente se obtuvo en 1949 y fue ejercido por primera vez en las elecciones presidenciales de 1952 (ibid).

Obtenido el derecho al sufragio, y al ser el eje central de articulación del movimiento, éste se desvaneció, dejando pendiente demandas de igualdad en el acceso a recursos sociales basado en género, por la renovada preponderancia de demandas de justicia social y económica que emergieron con fuerza en las décadas siguientes. Las demandas para transformar el orden de género y la situación de desventaja de las mujeres se debilitaron hasta desaparecer por las siguientes dos décadas y no reemergieron sino hasta fines de los 70, en medio de uno de los períodos más represivos de la historia de Chile (Kirkwood 1986). ¿Cuáles podrían ser las razones detrás del "silencio feminista" (Kirkwood, 1986) entre 1950 y 1970, si las condiciones de desventaja generadas por el limitado acceso de las mujeres a recursos sociales no evidenciaron transformaciones radicales en el período? La respuesta está vinculada a las condiciones que posibilitan el surgimiento de movimientos sociales capaces de desafiar el orden de género imperante. Evidentemente, una estructura de oportunidades, pero además, históricamente la identidad grupal surgida en el marco de la marginación sistemática de lo público, ha sido condición previa para la formación de alianzas y ha servido como preludio para la acción colectiva y movilización de mujeres.

La reemergencia de la movilización feminista después una larga ausencia estuvo ligada, en los años setenta, a la estructura de oportunidad abierta con la destrucción de la democracia electoral. El nuevo movimiento feminista estuvo ligado a partidos de izquierda y compartió el compromiso de transformar la esfera pública. Durante el régimen militar se produjo un realineamiento partidista que unificó a las fuerzas progresistas para enfrentar la dictadura. Las mujeres adquirieron una renovada legitimidad política a la luz de la exaltación de su rol en la refundación de la patria ideada por los militares en el poder. La lucha por la recuperación de la democracia proporcionó las condiciones políticas que hicieron posible vincularla con la lucha feminista: “democracia en el país y en la casa” avanzaba el eslogan de la época.

Una vez que el objetivo de derrotar la dictadura fue alcanzado, el elemento simbólico unificador de la lucha en común desapareció. El movimiento de mujeres fue afectado particularmente por la desarticulación que se produjo entre distintas organizaciones y actores de la sociedad civil y la merma de un objetivo político común, que dio pie al surgimiento de conflictos que se habían mantenido hasta ese entonces en segundo plano. El retorno de los partidos políticos afectó la oportunidad política de los movimientos sociales, en la medida que los desplazó de las negociaciones y del proceso político electoral y acentuó las diferencias entre ellos, tanto como al interior de ellos (Garretón 1993). En el caso del movimiento de mujeres, uno de los factores que produjo mayor conflicto fue la oposición entre aquellas que promovían el desarrollo de estrategias de acción en esferas autóno- 
mas del estado y sus instituciones, por una parte, y aquellas a favor de la participación de estos espacios institucionales para promover el cambio “desde adentro”, por otra (Ríos, Godoy y Guerrero 2004).

Además de estas divisiones respecto del tipo de estrategia global que debería seguir el movimiento de mujeres, durante y después del proceso de transición se sumaron quiebres de carácter político partidista entre distintos sectores. Se produjo una escisión entre las mujeres y organizaciones que se vincularon al proyecto político de la Concertación de Partidos por la Democracia, instalada en el poder desde 1990, y las que se identificaron con sectores de izquierda que se mantuvieron fuera de ese pacto político (Ríos, Godoy y Guerrero 2004).

La desarticulación del movimiento feminista en la década de los 90 fue coadyuvada por la repentina escasez de recursos económicos del exterior, fondos que comenzaron su retirada del país en este período, y por el nuevo contexto democrático que mantuvo las políticas de desarrollo con un sesgo neoliberal. Como varios autores han planteado (Schild, 1998; Álvarez, Dagnigno y Escobar, 1998), las políticas económicas que caracterizaron los cambios económicos durante y después de la dictadura militar, no sólo tuvieron un impacto en la redefinición del rol del estado y el mercado, sino que constituyeron el marco de profundos cambios culturales que se plasmaron en la comprensión de lo que conforma la sociedad civil. En este marco, sostiene Schild, la noción de ciudadanía se transformó en sinónimo de autonomía y desarrollo personal a través de relaciones con el mercado. En otras palabras, las dinámicas del mercado alcanzaron más allá del campo económico para atravesar todas las relaciones sociales (Larraín, 2005), determinando además importantes transformaciones en la noción de feminidad reflejada en las expectativas e ideales de género, imbuidos ambos de valores como la autovalencia y el individualismo (Mora, 2006).

Nos preguntamos entonces, ¿qué posibilidades de rearticulación de un movimiento feminista pueden visualizarse en el marco de la individualización de la sociedad civil, efecto argumentado de transformaciones económicas experimentadas por la sociedad chilena?; ¿Es concebible la reconstitución de un movimiento de mujeres, dada las profundas diferencias de enfoque y estrategia que facilitaron la fragmentación del mismo en los años 90?; ¿Es un movimiento la única -o la más efectiva- vía hacia la transformación del orden de género imperante?; Y ¿se han abierto potenciales estructuras de oportunidades en el actual escenario político? Abordamos estas preguntas a continuación.

\section{La Alternativa de una Política de Coalición}

Una de las principales paradojas enfrentadas por los movimientos sociales en Chile es, como sugieren distintos autores, el predominio de una lógica individualizadora en las últimas tres décadas, que ha acarreado un debilitamiento de la cohesión social, con efectos en la posibilidad de iden- 
tificar objetivos en común que se traduzcan en el surgimiento de una solidaridad colectiva. Esto crea un escenario cada vez más adverso para la conformación de un movimiento monolítico y de una identidad que lo represente (Beck, 1998.) ${ }^{1}$. En esta línea, Alvarez et al (2003), argumentan que el campo político cultural ha sido redefinido de tal manera que el lenguaje de los movimientos sociales no tiene capacidad de interpelación y que por tanto, han quedado al designio de otros agentes articuladores. Chile es, de acuerdo a estos autores, un caso paradigmático de despliegue de estrategias de ajuste social (programas sociales que hacen digerible el ajuste económico) que contribuyen a alterar las bases socio-culturales de la movilización política. De acuerdo a estos autores, la fragmentación que han introducido políticas de ajuste económico presenta una importante barrera al surgimiento de la política de representación.

En este sentido, la escisión del movimiento feminista post-dictadura, que tuvo como una de sus principales causas la adopción de estrategias antagónicas de incorporación al Estado y sus organismos, y por otro lado, de rechazo a la vinculación del movimiento con la mayoría de los intereses institucionalizados, puede también ser entendida como una división en torno al nuevo orden económico y social. Los programas sociales de acción paliativa no fueron apoyados unánimemente y junto con la incorporación de quienes fueron parte del movimiento en distintas esferas de gobierno, han tenido un efecto divisivo, previniendo el surgimiento de un movimiento representativo de las mujeres chilenas, más allá de solidaridades en torno a asuntos de género específicos.

Sin embargo, planteamos que este nuevo escenario no implica la desaparición de un campo de acción feminista. Sugerimos que la complejización del escenario político-social antes mencionada, no se traduce en la imposibilidad de la acción. En efecto, los movimientos sociales de antaño no han desaparecido, sino que se han diversificado para ocupar múltiples espacios de acción, con distintas estrategias, y con distintos tipos de expresiones organizacionales e institucionales (Ríos et al. 2004). Además de la protesta, los campos de acción se han extendido, por ejemplo, al "lobby" para afectar políticas públicas sensibles al género; a la generación de conocimiento a través de universidades y centros de investigación (que surgieron principalmente en la década de los 90); y al desarrollo de proyectos o programas vinculados al Estado u otros organismos nacionales e internacionales.

El despliegue de campos diferentes de acción feminista, ha repercutido ventajosamente en la incorporación de algunos temas de género en la agenda pública, abriendo debates legislativos y de opinión pública sobre asuntos tan relevantes como protección social y salud sexual y reproductiva. Sin embargo, esta diversificación ha incidido a la vez negativamente en la

\footnotetext{
${ }^{1}$ Aunque Beck considera que el fin de esta manera de hacer política podría sólo anticipar el comienzo de su reemplazo por una nueva lógica, articulada en torno a una sociedad mundial
} 
participación de organizaciones de base, la que se ha visto restringida principalmente por la escasez de recursos de información y financieros, así como por el nuevo énfasis en la profesionalización de quienes definen -o tienen el poder de influir- en la agenda pública de género.

Si el debilitamiento del movimiento feminista post-dictadura, en términos de su capacidad de movilización política, se debe en parte a la emergencia de distintos campos de acción y promoción de intereses en el ámbito jurídico, simbólico y material, ello constituye una barrera menor para la conformación de un movimiento feminista democrático. Por un lado, se podría argumentar que se han multiplicado las organizaciones de la sociedad civil que adoptan estrategias mixtas (participación en el Estado u otras instituciones, y a la vez, movilización), con potencial de ampliar e intensificar su influencia en el debate público. Por otro, la diversificación de las esferas de debate feminista no sólo tienen potencial democrático, sino que han expandido y profundizado la gama de temas considerados como "asuntos de género" en sus consecuencias.

$¿$ Constituyen estas nuevas condiciones el ocaso de los movimientos sociales? Como actor social que invoca la necesidad de una identidad política como eje de la acción, el surgimiento y permanencia de los movimientos está delimitado por las transformaciones sociales, políticas y económicas que ha experimentado el país en la última década. La historia del movimiento de mujeres en la última década refleja la fragilidad de la identidad política que lo sostenía, la que se hizo patente cuando el reconocimiento de intereses dispares se transformó en conflictivo. Y es que la política de la identidad implica siempre cierta violencia en la preeminencia de un fragmento que representa al todo. Como sostiene Butler (2001), la política de la representación refleja diferencias de poder en el predominio de una noción de "unidad” o de "diálogo", siendo plausible, y más bien probable, que lo que es definido como diálogo para el grupo sea entendido como exclusión por uno o muchos otros.

Esta paradoja de la representación queda evidenciada en las razones esgrimidas por las participantes del movimiento de mujeres para explicar su fragmentación y plantea la necesidad de repensar la política de representación, basada en una identidad, por una política de coalición. Tal política no debiera presuponer el contenido de la categoría “mujeres”, sino proponer "una serie de encuentros dialógicos en los que mujeres de posiciones diversas articulan distintas identidades dentro del marco de una coalición emergente” (Butler, 2001:47). Suponer que la categoría "mujeres" está siempre incompleta da pie para su permanente impugnación, a la vez que es posible perseguir el surgimiento de "unidades provisionales en el contexto de acciones concretas cuyos propósitos no son la articulación de identidad" (ibid: 48).

Tal aproximación a un movimiento de mujeres, abre la posibilidad de articular coaliciones en el nuevo escenario político-cultural del Chile post-dictadura. Ello se hace necesario porque, como sugerimos, la frag- 
mentación del movimiento se ha facilitado por una nueva lógica de acción individual, que puede ser vista como producto de la interpenetración de lógicas de mercado en las relaciones sociales. Pero además, se hace necesario por la intensificación de diferencias en el acceso a recursos, marcadas no sólo por las diferencias de clase social y las redes que ello conlleva, sino que por la mayor cercanía y facilidad de algunos segmentos para obtener fuentes de financiamiento estatal e internacional. Tanto la ardua historia del movimiento de mujeres en Chile como el actual contexto social, económico y político, hacen del objetivo de articular una identidad más una quimera que un proyecto realizable. La tarea consiste más bien, en promover encuentros dialógicos entre mujeres diferentemente posicionadas en torno a asuntos de género específicos.

¿Existen hoy potenciales aperturas en la estructura de oportunidades que posibiliten la emergencia de una política de coalición? En la siguiente sección, sugerimos que las condiciones generadas por el gobierno de Michelle Bachelet constituyen tal apertura.

\section{La Importancia del Gobierno Bachelet}

La elección de la Presidenta Michelle Bachelet en 2006 marcó un hito en la historia de la República, no sólo por ser la primera presidenta de Chile, sino también por su lejanía del establishment político y por su historia de persecución durante la dictadura militar. Varios factores contribuyeron al surgimiento de su figura en el escenario político: por una parte, el desgaste y las disrupciones que afectaban a la coalición gobernante abrió oportunidades para la inclusión de personas no pertenecientes a la elite política tradicional. Segundo, la presión por equidad de género ejercida desde la época de la dictadura militar, había llevado a mujeres a ocupar posiciones de liderazgo dentro de los gobiernos democráticos. Y, tercero, la popularidad y el apoyo con que Bachelet contaba entre las mujeres chilenas llevó a que por primera vez en la historia del país ellas votaran mayoritariamente por la izquierda (Power, 2004).

Bachelet se diferenció de los gobiernos concertacionistas que la precedieron en adoptar el tema de la equidad de género como uno de los ejes centrales de su gobierno. Es así como inauguró su mandato con un gabinete paritario, priorizó una reforma del sistema de pensiones con beneficios para mujeres dueñas de casa, amplió la red de cuidado infantil para los sectores más pobres, e introdujo la distribución de la anticoncepción de emergencia ${ }^{2}$, entre otras preocupaciones de género. Este intento decidido por avanzar en la agenda de derechos de las mujeres no tiene precedentes. Unido a la consistente alza de popularidad de la Presidenta ${ }^{3}$, a la mayor

\footnotetext{
${ }^{2}$ Medida impugnada exitosamente por el Tribunal Constitucional

${ }^{3}$ Traspasando la barrera del 70\% en la encuesta más influyente, en agosto de 2009. Encuesta CEP en www.cepchile.cl
} 
presencia de mujeres en esferas de poder, y a los cambios culturales que han ido aparejados a la administración Bachelet, proporcionan en conjunto una nueva estructura de oportunidades receptiva a la introducción de demandas de género y a la articulación de alianzas para traducirlas en políticas públicas.

La diversidad de "asuntos de género" que han entrado a la agenda pública y la resistencia que han encontrado entre las fuerzas conservadoras fuera y dentro de la coalición gobernante, hacen perentoria la identificación de los espacios y oportunidades abiertas, la acción conciente y la alianza concertada en torno a debates y demandas de "las mujeres". En la actualidad no hay duda que existen múltiples organizaciones y colectivos cuya estrategia es promover diálogos y alianzas entre mujeres. Se requiere por tanto de vincular la movilización feminista con procesos de democratización más amplios y de la existencia de un realineamiento de las alianzas político partidarias existentes en torno a las demandas de género. 


\section{Bibliografía}

Alvarez, S., Danigno, E., Escobar, A.(1998) "Introduction: The Cultural and the Political in Latin American Social Movements" en Cultures of Politics Politics of Cultures. Revisioning Latin American Social Movements, eds. Alvarez,S., Danigno, E., Escobar, A., Westview Press, Boulder.

Alvarez, S. (2003) "Encountering Latin American and Caribbean Feminisms." en Signs: Journal of Women in Culture and Society $\mathrm{N}^{\circ} 2$, EEUU

Beck, U., (1998) ¿Qué es la Globalización? Falacias del globalismo, respuestas a la globalización. Paidós, Barcelona.

Butler, J. (2001) El género en disputa, Paidós, Barcelona.

Cubillos, S. (1994) “Reflexiones en torno al accionar político del feminismo autónomo en Santiago de Chile. 1990-1993,” Ponencia presentada en el Segundo Foro Nacional Feminista. Movimiento Feminista Autónomo. Santiago.

Drake, P. (1978) Socialism and populism in Chile, 1932-52, University of Illinois Press, Urbana.

Encuesta CEP (2004). Consultado en Septiembre de 2009 en página web institucional del Centro de Estudios Públicos: http://www.cep.cl

Gamson,W y Meyer, D., (1996) “The Framing of Political Opportunity.” En Comparative Perspectives on Social Movements: Political Opportunities, Mobilizing Structures, and Cultural Framing, Cambridge University Press eds. Doug McAdam, John D. McCarthy, and Mayer N. Zald. Cambridge.

Garretón, M, (1993) “La oposición política y el sistema partidario en el régimen militar chileno: Un proceso de aprendizaje para la transición,” en El difícil camino hacia la democracia en Chile, 1982 - 1990, ed. Paul W. Drake and Ivan Jasksic. FLACSO, Santiago.

Kirkwood, J. (1986) Ser política en Chile: las feministas y los partidos, Facultad Latinoamericana de Ciencias Sociales, Santiago.

Larraín, J. (2005) ¿América Latina Moderna? Globalización e Identidad, LOM Santiago.

Lavrin, A. (1995) Women, feminism, and social change in Argentina, Chile, and Uruguay, 1890-1940, University of Nebraska Press, Lincoln.

McAdam, D., J. McCarthy y M. Zald. (1996), Comparative Perspectives on Social Movements: Political opportunities, mobilizing structures, and cultural framings. Cambridge University Press, Cambridge. 
Mora, C (2006) "The Meaning of the Womanhood in the Neoliberal Age: Class and Age-Based Narratives of Chilean Women” en Gender Issues, N ${ }^{\circ}$ 2, New York.

Power, M (2004) “More than mere pawns: Right- Wing Women in Chile”, en Journal of Women's History, $\mathrm{N}^{\mathrm{o}} 3$, EEUU.

Ríos, M., Godoy, L., Guerrero, E.,(2003)Un nuevo silencio feminista, Cuarto Propio, Santiago.

Ríos, M.,Godoy, L., y Guerrero, E., (2004) ¿Un Nuevo Silencio Feminista? La transformación de un movimiento social en el Chile postdictadura. Centro de Estudios de la Mujer. Santiago.

Schild, V., (1998), “New Subjects of Rights? Women's Movements and the Construction of Citizenship in the "New Democracies"”, en "Cultures of Politics Politics of Cultures. Revisioning Latin American Social Movements” eds. Alvarez,S., Danigno, E., Escobar, A., Westview Press, Boulder.

Tarrow, S. (1998) Power in Movement: Social movements and contentious politics. 2th ed.Cambridge University Press, Cambridge.

Valenzuela, M. y Mora, C. (2009) "El trabajo doméstico como actividad económica y laboral” en Trabajo doméstico y equidad de género en Latinoamérica: desafíos para el trabajo decente. Eds. María Elena Valenzuela y Claudia Mora. En Prensa. OIT, Santiago. 